\title{
Refinement of Representation Theorems for Context-Free
}

\section{Languages*}

\author{
Kaoru FUJIOKA ${ }^{\dagger a)}$, Member
}

\begin{abstract}
SUMMARY In this paper, we obtain some refinement of representation theorems for context-free languages by using Dyck languages, insertion systems, strictly locally testable languages, and morphisms. For instance, we improved the Chomsky-Schützenberger representation theorem and show that each context-free language $L$ can be represented in the form $L=h(D \cap R)$, where $D$ is a Dyck language, $R$ is a strictly 3-testable language, and $h$ is a morphism. A similar representation for context-free languages can be obtained, using insertion systems of weight $(3,0)$ and strictly 4-testable languages.

key words: Chomsky-Schützenberger representation theorem, context-free languages
\end{abstract}

\section{Introduction}

Representing a class of languages through operations on its subclasses is a traditional topic within formal language theory. We focus on characterizing context-free languages by using insertion systems together with some additional mechanisms.

A traditional topic in insertion systems in which we can use only insertion operations has been the representation of the class of recursively enumerable languages by simple operations on a small subclass of it [1]. From the definition of insertion operations, one would easily imagine that by using only insertion operations, we generate only contextsensitive languages.

It has been shown that using insertion systems together with some morphisms, characterizing recursively enumerable languages is accomplished in [2], [3]. In [4], each recursively enumerable language $L$ can be represented in a similar way to the well-known Chomsky-Schützenberger representation of context-free languages, i.e. $L=h(L(\gamma) \cap$ $D$ ), where $\gamma$ is an insertion system, $h$ is a projection, and $D$ is a Dyck language. They also characterize the class of context-free languages using insertion systems of weight $(3,0)$ and star languages.

On the other hand, the class of strictly locally testable languages is known as a proper subclass of regular language classes. The equivalence relation between a certain type of splicing languages and strictly locally testable languages is known [5]. In this paper, we make use of strictly locally

\footnotetext{
Manuscript received March 20, 2009.

Manuscript revised July 12, 2009.

${ }^{\dagger}$ The author is with the Office for Strategic Research Planning, Kyushu University, Fukuoka-shi, 812-8581 Japan.

*This is an extended version of the presentation at LA Symposium 2008

a)E-mail: kaoru@tcslab.csce.kyushu-u.ac.jp

DOI: 10.1587/transinf.E93.D.227
}

testable languages as the additional mechanisms for characterizing context-free languages.

In insertion systems, a pair of the maximum length of inserted strings and the one of context-checking strings, called weight is an important parameter for generative powers. As for strictly locally testable languages, the length of local testability-checking is considered.

We prove that each context-free language $L$ can be represented in the form $L=h(D \cap R)$, where $D$ is a Dyck language, $h$ is a morphism, and $R$ is a strictly 3 -testable language. A similar representation for context-free languages can be obtained, using insertion systems of weight $(3,0)$ and strictly 4-testable languages. The optimality of these results, in terms of weight in insertion operations and the parameter of strictly locally testable languages remains to be checked.

\section{Preliminaries}

In this section, we introduce necessary notation and basic definitions needed in this paper. We assume the reader to be familiar with the rudiments on basic notions in formal language theory (see, e.g., [2], [6]).

\subsection{Basic Definitions}

For an alphabet $V, V^{*}$ is the set of all strings of symbols from $V$ which includes the empty string $\lambda$. For a string $x \in V^{*}$, $|x|$ denotes the length of $x$. Furthermore, $|x|_{V^{\prime}}$ represents the number of occurrences of the symbols of the set $V^{\prime}$ in $x$. For $0 \leq k \leq|x|$, let $\operatorname{Pre}_{k}(x)$ and $S u f_{k}(x)$ be the prefix and the suffix of $x$ of length $k$, respectively. For $0 \leq k \leq|x|$, let $\operatorname{Int}_{k}(x)$ be the set of proper interior substrings of $x$ of length $k$, while if $|x|=k$ then $\operatorname{Int}_{k}(x)=\emptyset$.

\subsection{Normal Forms of Grammars}

A phrase structure grammar is a quadruple $G=(N, T, P, S)$, where $N$ is a set of nonterminal symbols, $T$ is a set of terminal symbols, $P$ is a set of production rules, and $S$ in $N$ is the initial symbol. A rule in $P$ is of the form $r: \alpha \rightarrow \beta$, where $\alpha \in(N \cup T)^{*} N(N \cup T)^{*}, \beta \in(N \cup T)^{*}$, and $r$ is a label such that there are no production rules with the same label, $\operatorname{Lab}(P)=\{r \mid r: \alpha \rightarrow \beta \in P\}$. We often omit labels of production rules, if not necessary. For any $x$ and $y$ in $(N \cup T)^{*}$, if $x=u \alpha v, y=u \beta v$, and $r: \alpha \rightarrow \beta \in P$, then we write

$$
x \stackrel{r}{\Longrightarrow} G \text {. }
$$


We say that $x$ directly derives $y$ with respect to $G$. If there is no confusion, we write $x \Longrightarrow y$. The reflexive and transitive closure of $\Longrightarrow$ is denoted by $\Longrightarrow^{*}$. A derivation of length $k \geq 0$ from $x$ to $y$ is a sequence $\left(x_{0}, x_{1}, \cdots, x_{k}\right)$ of strings in $(N \cup T)^{*}$ such that $x_{i-1} \Longrightarrow x_{i}$ for $i=1, \cdots, k$ and $x=x_{0}$, $y=x_{k}$, which is denoted by $x \Longrightarrow^{k} y$.

We define a language $L(G)$ generated by a grammar $G$ as follows:

$$
L(G)=\left\{w \in T^{*} \mid S \Longrightarrow{ }_{G}^{*} w\right\} .
$$

It is well known that the class of languages generated by the phrase structure grammars is equal to the class of recursively enumerable languages $R E[6]$.

A grammar $G=(N, T, P, S)$ is context-free if $P$ is a finite set of context-free rules of the form $A \rightarrow \alpha$, where $A \in$ $N$ and $\alpha \in(N \cup T)^{*}$. A language $L$ is a context-free language if there is a context-free grammar $G$ such that $L=L(G)$. Let $C F$ be the class of context-free languages.

A context-free grammar $G=(N, T, P, S)$ is in Chomsky normal form if each production rule in $P$ is of one of the following forms:

1. $X \rightarrow Y Z$, where $X, Y, Z \in N$.

2. $X \rightarrow a$, where $X \in N, a \in T$.

3. $S \rightarrow \lambda$ (only if $S$ does not appear in right-hand sides of rules).

It is well known that, for each context-free language $L$, there is a context-free grammar in Chomsky normal form generating $L[6]$.

For an alphabet $\Sigma=\left\{a_{1}, \cdots, a_{n}\right\}$ with $n \geq 1$, let $\bar{\Sigma}=\left\{\bar{a}_{1}, \cdots, \bar{a}_{n}\right\}$ be an alphabet such that for each $1 \leq i \leq n$, $\bar{a}_{i}$ is a barred copy of $a_{i}$. $\quad \Sigma$ and $\bar{\Sigma}$ are considered to be disjoint. Then Dyck language $D$ over $\Sigma$ and $\bar{\Sigma}$ is defined to be the context-free language generated by the grammar $G_{D}=(\{S\}, \Sigma \cup \bar{\Sigma}, P, S)$, where $P=\{S \rightarrow S S, S \rightarrow$ $a S \bar{a}, S \rightarrow \lambda \mid a \in \Sigma, \bar{a} \in \bar{\Sigma}$. Let $D y c k$ be a class of Dyck languages.

A grammar $G=(N, T, P, S)$ is regular if $P$ is a finite set of rules of the form $X \rightarrow \alpha$, where $X \in N$ and $\alpha \in$ $T N \cup T \cup\{\lambda\}$. A language $L$ is a regular language if there is a regular grammar $G$ such that $L=L(G)$. Let $R E G$ be the class of regular languages.

We are going to define a strictly locally testable language, which is one of the main objectives of the present work.

Let $k$ be a positive integer. A language $L$ over $T$ is strictly $k$-testable if there is a triplet $S_{k}=(A, B, C)$ with $A, B, C \subseteq T^{k}$ such that for any $w$ with $|w| \geq k, w$ is in $L$ iff $\operatorname{Pre}_{k}(w) \in A, \operatorname{Suf}_{k}(w) \in B, \operatorname{Int}_{k}(w) \subseteq C$.

Note that if $L$ is strictly $k$-testable, then $L$ is strictly $k^{\prime}$-testable for all $k^{\prime}>k$. Further, the definition of strictly $k$-testable says nothing about the strings of "length $k-1$ or less".
A language $L$ is strictly locally testable iff there exists an integer $k \geq 1$ such that $L$ is strictly $k$-testable. Let $L O C(k)$ be the class of strictly $k$-testable languages. Then one can prove the following theorem.

Theorem 1: [7] $\operatorname{LOC}(1) \subset \operatorname{LOC}(2) \subset \cdots \subset \operatorname{LOC}(k) \subset$ $\cdots \subset L O C \subset R E G$.

We are now going to define an insertion system. An insertion system is a triple $\gamma=\left(T, P, A_{X}\right)$, where $T$ is an alphabet, $P$ is a finite set of insertion rules of the form $r$ : $(u, x, v)$ with $u, x, v \in T^{*}, A_{X}$ is a finite set of strings over $T$ called axioms, and $r$ is a label such that there are no insertion rules with the same label, $\operatorname{Lab}(P)=\{r \mid r:(u, x, v) \in P\}$.

We write $\alpha \stackrel{r}{\Longrightarrow} \beta$ if $\alpha=\alpha_{1} u v \alpha_{2}$ and $\beta=\alpha_{1} u x v \alpha_{2}$ for some insertion rule $r:(u, x, v) \in P$ with $\alpha_{1}, \alpha_{2} \in T^{*}$. If there is no confusion, we write $\alpha \Longrightarrow \beta$. The reflexive and transitive closure of $\Longrightarrow$ is defined by $\Longrightarrow$.

A language generated by $\gamma$ is defined by

$$
L(\gamma)=\left\{w \in T^{*} \mid s \Longrightarrow_{\gamma}^{*} w \text {, for some } s \in A_{X}\right\} .
$$

An insertion system $\gamma=\left(T, P, A_{X}\right)$ is said to be of weight $(m, n)$ if

$$
\begin{aligned}
& m=\max \{|x| \mid(u, x, v) \in P\}, \\
& n=\max \{|u| \mid(u, x, v) \in P \text { or }(v, x, u) \in P\} .
\end{aligned}
$$

For $m, n \geq 0, I N S_{m}^{n}$ denotes the class of all languages generated by insertion systems of weight $\left(m^{\prime}, n^{\prime}\right)$ with $m^{\prime} \leq m$ and $n^{\prime} \leq n$. When the parameter is not bounded, we replace $m$ or $n$ with $*$.

For insertion systems, there exist the following results.

Theorem 2 ([2]): 1 . For the class of finite languages $F I N$ and the class of context-sensitive languages $C S$, $F I N \subset I N S_{*}^{0} \subset I N S_{*}^{1} \cdots \subset I N S_{*}^{*} \subset C S$.

2. $R E G \subset I N S_{*}^{*}$.

3. $I N S_{*}^{1} \subset C F$.

4. CF is incomparable with all $I N S_{*}^{n}(n \geq 2)$, and $I N S_{*}^{*}$.

5. INS 2 contains non-semilinear languages.

A mapping $h: V^{*} \rightarrow T^{*}$ is called morphism if $h(\lambda)=\lambda$ and $h(x y)=h(x) h(y)$ for any $x, y \in V^{*}$. For languages $L_{1}$, $L_{2}$, and morphism $h$, we use the following notation: $h\left(L_{1} \cap\right.$ $\left.L_{2}\right)=\left\{h(w) \mid w \in L_{1} \cap L_{2}\right\}$. For language classes $\mathcal{L}_{1}$ and $\mathcal{L}_{2}$, we introduce the following class of languages:

$$
\begin{aligned}
& H\left(\mathcal{L}_{1} \cap \mathcal{L}_{2}\right) \\
& \quad=\left\{h\left(L_{1} \cap L_{2}\right) \mid h \text { is a morphism, } L_{i} \in \mathcal{L}_{i}(i=1,2)\right\} .
\end{aligned}
$$

\section{Modifications of Păun's Theorem}

We will show how context-free languages can be characterized by insertion systems and strictly locally testable languages. In some respect the proof technique from [4] might be helpful to follow the main proof of this section, in which star languages are used instead of strictly locally testable languages. 
Lemma 1: $C F \subseteq H\left(I N S_{3}^{0} \cap L O C(4)\right)$.

Construction: Consider a context-free grammar $G=$ $(N, T, P, S)$ in Chomsky normal form. We construct an insertion system $\gamma=\left(\Sigma, P_{\gamma},\{S\}\right)$, where

$$
\begin{aligned}
V= & N \cup \operatorname{Lab}(P), \\
\Sigma= & V \cup \bar{V} \cup T, \\
P_{\gamma}= & \{(\lambda, Y Z r, \lambda),(\lambda, \bar{X} \bar{r}, \lambda) \mid r: X \rightarrow Y Z \in P\} \cup \\
& \{(\lambda, a r, \lambda),(\lambda, \bar{X} \bar{r}, \lambda) \mid r: X \rightarrow a \in P\} \cup \\
& \{(\lambda, r, \lambda),(\lambda, \bar{S} \bar{r}, \lambda) \mid r: S \rightarrow \lambda \in P\} .
\end{aligned}
$$

For the rule $r: X \rightarrow \alpha$ in $P$, we say that the two insertion rules $(\lambda, \alpha r, \lambda)$ and $(\lambda, \bar{X} \bar{r}, \lambda)$ in $P_{\gamma}$ are $r$-pair.

We define the projection $h: \Sigma^{*} \rightarrow T^{*}$ by

$$
\begin{array}{ll}
h(\lambda)=\lambda, & \\
h(\text { ax })=a h(x) & \text { if } a \in T, \\
h(a x)=h(x) & \text { otherwise. }
\end{array}
$$
where

Consider $R=A \Sigma^{*} \cap \Sigma^{*} B-\Sigma^{+} C^{\prime} \Sigma^{+}$with $C^{\prime}=\Sigma^{4}-C$,

$$
\begin{aligned}
A= & \{a r X \bar{X} \mid r: X \rightarrow a \in P\} \cup \\
& \{r S \bar{S} \bar{r} \mid r: S \rightarrow \lambda \in P\}, \\
B= & \left\{r S \bar{S} \bar{r} \mid r: S \rightarrow \alpha \in P, \alpha \in(N \cup T)^{*}\right\}, \\
C= & \left\{r X \bar{X} \bar{r}, X \bar{X} \bar{r} a, X \bar{X} \bar{r} r_{1}, \bar{X} \bar{r} a r_{1},\right. \\
& \bar{X} \bar{r} r_{1} Y, \overline{r_{1}} a r X, \overline{r_{1}} r X \bar{X}, \operatorname{arX} \bar{X} \mid \\
& r: X \rightarrow \alpha \in P, r_{1}: Y \rightarrow \alpha_{1} \in P, a \in T, \\
& \left.\alpha, \alpha_{1} \in(N \cup T)^{*}\right\} .
\end{aligned}
$$

Then $R$ is a strictly 4-testable language prescribed by $S_{4}=$ $(A, B, C)$. The language $R$ can be characterized by using

$$
\Omega=\left\{r X \bar{X} \bar{r} \mid r: X \rightarrow \alpha \in P, \alpha \in(N \cup T)^{*}\right\}
$$

such that $R \subset(\Omega \cup T \Omega)^{*}(B \cup T B)$. A nonterminal symbol $X$ in $r X \bar{X} \bar{r} \in \Omega$ is said to be $\Omega$-blocked. Intuitively, an $\Omega$ blocked nonterminal symbol $X$ in $r X \bar{X} \bar{r}$ means that $X$ has been used for the rule $r$.

Further, based on $\gamma$ and $R$, we define the followings: for each $X \in N$, let

$$
\gamma_{X}=\left(\Sigma, P_{\gamma},\{X\}\right)
$$

be an insertion grammar, and let

$$
R_{X}=A \Sigma^{*} \cap \Sigma^{*} B_{X}-\Sigma^{+} C^{\prime} \Sigma^{*}
$$

be a strictly 4-testable language, where $B_{X}=\{r X \bar{X} \bar{r} \mid r$ : $\left.X \rightarrow \alpha \in P, \alpha \in(N \cup T)^{*}\right\}$. There is a slight note on the form of $\Sigma^{+} C^{\prime} \Sigma^{*}$ in $R_{X}$. Then $R_{X}$ can be characterized by $R_{X} \subset(\Omega \cup T \Omega)^{*}$. For the case $X=S, \gamma_{S}=\gamma$ and $B_{S}=B$ hold, which implies $R_{X}=R$ from the definitions of $R_{X}$ and $R$.

Idea of the Proof: Since the labels of production rules $L a b(P)$ precisely identify an $r$-pair insertion rules and $R \subset$ $(\Omega \cup T \Omega)^{*}(B \cup T B)$ holds, a production rule $r$ in $P$ is simulated by $r$-pair insertion rules in $P_{\gamma}$. Furthermore, any successful derivation of $\gamma$ requires the use of $r$-pair insertion rules in which any consecutive (odd, even) derivations steps simulate one production $r$ in $G$. In a successful derivation, a nonterminal symbol $X$ in $r X \bar{X} \bar{r} \in \Omega$ implies that $X$ has been used for the rule $r$. Finally, the symbol in $\Sigma-T$ which is not used in $L(G)$ is eventually eliminated by the projection $h$.

To show $L(G)=h(L(\gamma) \cap R)$, we will show the following inclusion.

$[\boldsymbol{L}(\boldsymbol{G}) \subseteq \boldsymbol{h}(\boldsymbol{L}(\gamma) \cap \boldsymbol{R})]$ We will show that for any $X$ in $N$, if there is a derivation $X{\stackrel{r_{1} \cdots r_{n}}{\Longrightarrow}}_{G} a_{1} \cdots a_{l}$ with $a_{i} \in T(1 \leq i \leq l)$ then there is a string $w=a_{1} u_{1} \cdots a_{l} u_{l}$ in $L\left(\gamma_{X}\right) \cap R_{X}$, where

- for $l \geq 2, u_{i} \in \Omega^{+}(1 \leq i \leq l-1)$, and $u_{l} \in \Omega^{*}\left\{r_{1} X \bar{X} \bar{r}_{1}\right\}$,

- for $l=1, u_{1} \in \Omega^{*}\left\{r_{1} X \bar{X} \bar{r}_{1}\right\}$,

by induction on the length $n$ of derivations in $G$.

Base step: Suppose that there is a derivation $X \stackrel{r}{\Longrightarrow} a$. From the definition of $P_{\gamma}, r$-pair insertion rules $(\lambda, a r, \lambda)$ and $(\lambda, \bar{X} \bar{r}, \lambda)$ are in $P_{\gamma}$. Therefore, $X \Longrightarrow_{\gamma_{X}} X \bar{X} \bar{r} \Longrightarrow_{\gamma_{X}} \operatorname{ar} X \bar{X} \bar{r}$, where $\operatorname{ar} X \bar{X} \bar{r}$ is in $R_{X}$.

Induction step: We suppose that the claim holds for any $k \leq n$.

Consider a derivation $X \stackrel{r}{\Longrightarrow} \quad Y Z \stackrel{r_{1} \cdots r_{n}}{\Longrightarrow} a_{G} \quad a_{1} \ldots$ $a_{h} a_{h+1} \cdots a_{l} \in T^{+}$, where $l \geq 2, Y \Longrightarrow_{G}^{*} a_{1} \cdots a_{h}, Z \Longrightarrow_{G}^{*}$ $a_{h+1} \cdots a_{l}$, and $a_{i} \in T$ for $1 \leq i \leq l$. For the rule $r: X \rightarrow$ $Y Z$ in $P$, from the definition of $P_{\gamma}, r$-pair insertion rules $(\lambda, Y Z r, \lambda)$ and $(\lambda, \bar{X} \bar{r}, \lambda)$ are in $P_{\gamma}$. From the induction hypothesis, there are strings $a_{1} u_{1} \cdots a_{h} u_{h}$ and $a_{h+1} u_{h+1} \cdots a_{l} u_{l}$ such that $a_{1} u_{1} \cdots a_{h} u_{h} \in L\left(\gamma_{Y}\right) \cap R_{Y}, a_{h+1} u_{h+1} \cdots a_{l} u_{l} \in$ $L\left(\gamma_{Z}\right) \cap R_{Z}$, and $u_{i} \in \Omega^{+}$for $1 \leq i \leq l$. Therefore, there is a derivation

$$
\begin{aligned}
X & \Longrightarrow \gamma \\
& \Longrightarrow \bar{X} \bar{r} \Longrightarrow{ }_{\gamma}^{*} a_{1} u_{1} \cdots a_{h} u_{h} a_{h+1} \cdots a_{l} \bar{X}_{l} r \\
&
\end{aligned}
$$

The fact $w$ is in $R_{X}$ can be derived from the definitions of $R_{X}, R_{Y}$, and $R_{Z}$. For the projection $h, h(w)=a_{1} \cdots a_{l}$ holds. Therefore, for a string $w^{\prime}$ with $X \Longrightarrow_{G}^{*} w^{\prime}$, there exists a string $w$ such that $w \in L\left(\gamma_{X}\right) \cap R_{X}$ and $h(w)=w^{\prime}$.

In the above claim, consider the case $X=S$, then $\gamma_{S}=$ $\gamma$ and $R_{S}=R$ hold. Therefore, if a string $w^{\prime}$ is in $L(G)$, then there is a string $w$ such that $w \in L(\gamma) \cap R$ and $h(w)=w^{\prime}$.

Conversely, we consider the reverse inclusion.

$[\boldsymbol{L}(\boldsymbol{G}) \supseteq \boldsymbol{h}(\boldsymbol{L}(\gamma) \cap \boldsymbol{R})]$ We will show that for nonempty string $w$ in $L(\gamma) \cap R, h(w)$ is in $L(G)$. We start by showing that if a string $w$ is in $L\left(\gamma_{X}\right) \cap R_{X}$, then there is a derivation $X \Longrightarrow_{G}^{*} h(w)$.

Suppose that $w$ is in $L\left(\gamma_{X}\right) \cap R_{X}$. In order to derive the string $w$ in $R_{X} \subset(\Omega \cup T \Omega)^{*}$, without loss of generality, we may assume here that for each $r$ in $P$ the first two steps in $\gamma_{X}$ are performed by $r$-pair insertion rules. For the derivation $X \Longrightarrow{ }_{\gamma_{X}}^{2 n} w$, we will prove the claim by induction on $n$.

Base step: Suppose that there is a derivation $X \Longrightarrow_{\gamma_{X}}$ $X \bar{X} \bar{r} \Longrightarrow \gamma_{X} \operatorname{ar} X \bar{X} \bar{r} \in L\left(\gamma_{X}\right) \cap R_{X}$. From the definition of $P_{\gamma}$, for the $r$-pair insertion rules there is a production rule $r: X \rightarrow a$ in $P$. Then, there is a derivation $X \Longrightarrow_{G} a$, where $h(\operatorname{ar} X \bar{X} \bar{r})=a$.

Induction step: We suppose that the claim holds for 
any $k \leq n$. Consider a derivation $X \Longrightarrow_{\gamma_{X}} X \bar{X} \bar{r} \Longrightarrow{ }_{\gamma_{X}}$ $Y Z r X \bar{X} \bar{r} \Longrightarrow \gamma_{\gamma_{X}}^{2 n} w$, where $w \in L\left(\gamma_{X}\right) \cap R_{X}$.

For the $r$-pair insertion rules $(\lambda, Y Z r, \lambda)$ and $(\lambda, \bar{X} \bar{r}, \lambda)$, by the construction of $P_{\gamma}$, there is the production rule $r$ : $X \rightarrow Y Z$ in $P$.

For $w$ to be in $R_{X}$, considering the $r$-pair insertion rules in $\gamma_{X}, w$ is in the form of $u_{Y} u_{Z} r X \bar{X} \bar{r}$, where $u_{Y} u_{Z} \in \Sigma^{*}$. Further, the string $u_{Y} u_{Z}$ should be in $(\Omega \cup T \Omega)^{*}$, which implies that the symbols $Y$ and $Z$ must be $\Omega$-blocked. Then $r_{Y}$-pair insertion rules $\left(\left(\lambda, \alpha r_{Y}, \lambda\right),\left(\lambda, \bar{Y} \bar{r}_{Y}, \lambda\right)\right)$ and $r_{Z}$-pair of insertion rules $\left(\left(\lambda, \beta r_{Z}, \lambda\right),\left(\lambda, \bar{Z} \bar{r}_{Z}, \lambda\right)\right)$ with $\alpha, \beta \in(N \cup T)^{*}$ are used for $Y$ and $Z$ to be $\Omega$-blocked, respectively. We thus obtain $\alpha r_{Y} Y \bar{Y} \bar{r}_{Y}$ and $\beta r_{Z} Z \bar{Z} \bar{r}_{Z}$, respectively.

Then, let $u_{Y}=u_{1} r_{Y} Y \bar{Y} \bar{r}_{Y} \in(\Omega \cup T \Omega)^{*}$ and $u_{Z}=$ $u_{2} r_{Z} Z \bar{Z} \bar{r}_{Z} \in(\Omega \cup T \Omega)^{*}$ with $u_{1}, u_{2} \in \Sigma^{*}$. Since $w=$ $u_{Y} u_{Z} r X \bar{X} \bar{r}$ is in $R_{X}, u_{Y} \in R_{Y}$ and $u_{Z} \in R_{Z}$ hold from the definition of $R_{Y}$ and $R_{Z}$. Further, from the assumption that $w$ is in $L\left(\gamma_{X}\right), u_{Y}$ is in $L\left(\gamma_{Y}\right)$ and $u_{Z}$ is in $L\left(\gamma_{Z}\right)$. As a result, from the induction hypothesis, there are derivations $Y \Longrightarrow{ }_{G}^{*} h\left(u_{Y}\right), Z \Longrightarrow_{G}^{*} h\left(u_{Z}\right)$.

Therefore, there is a derivation

$$
\begin{aligned}
X & \Longrightarrow_{G} Y Z \\
& \Longrightarrow{ }_{G}^{*} h\left(u_{Y}\right) h\left(u_{Z}\right)=h\left(u_{Y} u_{Z} r X \bar{X} \bar{r}\right)=h(w) .
\end{aligned}
$$

In the above claim, consider the case $X=S$, then $\gamma_{S}=\gamma$ and $R_{X}=R$ hold. Therefore, if there is a nonempty string $w \in L(\gamma) \cap R$, then a string $h(w)$ is in $L(G)$.

Proof of Lemma 1: Let us consider the case that $\lambda$ is in $L(G)$. Since $G$ is in Chomsky normal form, $\lambda$ is in $L(G)$ if and only if for $\lambda$ there is a derivation $S \stackrel{r}{\Longrightarrow} \lambda$. By the construction of $P_{\gamma}$ and $R$, the string $\lambda$ is in $L(G)$ if and only if $(\lambda, r, \lambda),(\lambda, \bar{S} \bar{r}, \lambda) \in P_{\gamma}$ and $r S \bar{S} \bar{r} \in A \cap B$. Then there is a derivation $S \Longrightarrow \gamma S \bar{S} \bar{r} \Longrightarrow \gamma r S \bar{S} \bar{r} \in A \cap B$. From the definition of $h, h(r S \bar{S} \bar{r})=\lambda$. Therefore, $\lambda$ is in $L(G)$ if and only if $\lambda$ is in $h(L(\gamma) \cap R)$. Together with the inclusions $L(G) \subseteq h(L(\gamma) \cap R)$ and $L(G) \supseteq h(L(\gamma) \cap R)$, we complete the proof.

From Theorem 2, the class of context-free languages includes the class of insertion languages $I N S_{3}^{0}$. Together with the fact that the class of context-free languages is closed under intersection with regular languages and morphisms, we have the following lemma.

Lemma 2: $H\left(I N S_{3}^{0} \cap L O C(4)\right) \subseteq C F$.

From Lemma 1 and Lemma 2, we have the following theorem.

Theorem 3: $C F=H\left(I N S_{3}^{0} \cap L O C(4)\right)$.

Furthermore, from the fact that for arbitrary $k$ with $k \geq$ 1 , the class of regular languages includes the class of strictly $k$-testable languages, we have the following corollary.

Corollary 1: $C F=H\left(I N S_{3}^{0} \cap \operatorname{LOC}(k)\right)(k \geq 4)$.

\section{Refinement of the Chomsky-Schützenberger Repre- sentation Theorem}

In this section, we improve the celebrated ChomskySchützenberger representation theorem for the class of context-free languages, in the form $C F=H(D y c k \cap R E G)$. To show the equality $C F=H(D y c k \cap L O C(3))$, we first consider the following theorem.

Theorem 4: $H(D y c k \cap R E G) \subseteq H(D y c k \cap L O C(3))$.

Construction: Let $h: T^{*} \rightarrow \Gamma^{*}$ be a morphism, $D$ be Dyck language over $\Sigma$ and $\bar{\Sigma}$, and $G=(N, T, P, S)$ with $T=$ $\Sigma \cup \bar{\Sigma}$ be a regular grammar. We construct Dyck language $D^{\prime}$, strictly 3-testable languages, and morphism $h^{\prime}$ as follows.

- Strictly 3-testable languages.

For any $N_{1}, N_{2} \in N$, we construct

$$
L\left(N_{1}: N_{2}\right)=A\left(N_{1}\right) \Sigma^{*} \cap \Sigma^{*} B\left(N_{2}\right)-\Sigma^{+} C^{\prime} \Sigma^{+}
$$

with $C^{\prime}=\Sigma^{3}-C$, where

$$
\begin{aligned}
A\left(N_{1}\right)= & \left\{N_{1} \bar{N}_{1} a \mid N_{1} \rightarrow a X \in P, X \in N, a \in T\right\}, \\
B\left(N_{2}\right)= & \left\{a N_{2} \bar{N}_{2} \mid X \rightarrow a N_{2} \in P, X \in N, a \in T\right\}, \\
C= & \{\bar{X} a Y, a Y \bar{Y}, X \bar{X} a \mid X \rightarrow a Y \in P, \\
& X, Y \in N, a \in T\} .
\end{aligned}
$$

The language $L\left(N_{1}: N_{2}\right)$ can be characterized by using $\Gamma=T\{M \bar{M} \mid M \in N\}$ such that $L\left(N_{1}: N_{2}\right) \subseteq$ $\left\{N_{1} \bar{N}_{1}\right\} \Gamma^{*} T\left\{N_{2} \bar{N}_{2}\right\}$.

By using the new symbols $F$ and $\bar{F}$, for any $N_{1} \in N$, we construct

$$
\begin{aligned}
& L\left(N_{1}: F\right) \\
& \quad=A\left(N_{1}: F\right) \Sigma^{*} \cap \Sigma^{*} B\left(N_{1}: F\right)-\Sigma^{+} C^{\prime}(F) \Sigma^{+}
\end{aligned}
$$

with $C^{\prime}(F)=\Sigma^{3}-C(F)$, where

$$
\begin{aligned}
A\left(N_{1}: F\right)= & \left\{N_{1} \bar{N}_{1} a \mid N_{1} \rightarrow a X \in P\right. \text { or } \\
& \left.N_{1} \rightarrow a \in P, a \in T, X \in N\right\} \cup \\
& \left\{S \bar{S} F \mid N_{1}=S, S \rightarrow \lambda \in P\right\}, \\
B\left(N_{1}: F\right)= & \{a F \bar{F} \mid X \rightarrow a \in P, a \in T\} \cup \\
& \left\{S F \bar{F} \mid N_{1}=S, S \rightarrow \lambda \in P\right\}, \\
C(F)= & \{\bar{X} a Y, a Y \bar{Y}, X \bar{X} a \mid X \rightarrow a Y \in P, \\
& X, Y \in N, a \in T\} \cup \\
& \{X \bar{X} a, \bar{X} a F \mid X \rightarrow a \in P, \\
& X \in N, a \in T\} .
\end{aligned}
$$

From the above definitions, for any $N_{1} \in N$, the followings hold:

$$
\begin{aligned}
& A\left(N_{1}\right), B\left(N_{1}\right) \subset C \subset C(F), \\
& A\left(N_{1}\right) \subseteq A\left(N_{1}: F\right) .
\end{aligned}
$$

- Dyck language $D^{\prime}$.

By using the new symbols $F$ and $\bar{F}, D^{\prime}$ is a Dyck language over $\Sigma \cup N \cup\{F\}$ and $\bar{\Sigma} \cup \bar{N} \cup\{\bar{F}\}$.

- Homomorphism $h^{\prime}$.

For $V=T \cup N \cup \bar{N} \cup\{F, \bar{F}\}$, we define $h^{\prime}: V^{*} \rightarrow \Gamma^{*}$ by

$$
\begin{array}{ll}
h^{\prime}(\lambda)=\lambda & \\
h^{\prime}(a x)=h^{\prime}(a) h^{\prime}(x) & \text { if } a \in T, \\
h^{\prime}(a x)=h^{\prime}(x) & \text { if } a \notin T .
\end{array}
$$


Idea of the Proof: For any string $x$ in $L(G)$, a derivation $S \Longrightarrow_{G}^{*} x$ corresponds to a string $y \in D^{\prime} \cap L(S: F)$. Since Dyck language $D^{\prime}$ is defined over $\Sigma \cup N \cup F$ and $\bar{\Sigma} \cup \bar{N} \cup \bar{F}$, for Dyck language $D$ over $\Sigma$ and $\bar{\Sigma}$, any string $x$ in $D \cap L(G)$ is simulated by $D^{\prime} \cap L\left(G^{\prime}\right)$. Finally, the symbol in $N \cup \bar{N} \cup\{F, \bar{F}\}$ is eliminated and the symbol in $T$ is transformed into $\Gamma$ by $h^{\prime}$.

To show $h(D \cap L(G))=h^{\prime}\left(D^{\prime} \cap L(S: F)\right)$, we consider the following inclusion.

$\left[\boldsymbol{h}(D \cap \boldsymbol{L}(\boldsymbol{G})) \subseteq \boldsymbol{h}^{\prime}\left(D^{\prime} \cap \boldsymbol{L}(\boldsymbol{S}: \boldsymbol{F})\right)\right]$ To show the inclusion, we first prove that for any $x$ which satisfies that $x \in D,|x|=$ $2 n$, and $X \Longrightarrow_{G}^{*} x Y$ with $X, Y \in N$, there is a string $y \in$ $D^{\prime} \cap L(X: Y)$ such that $h^{\prime}(y)=h(x)$ by induction on $n$.

Base step: Consider a string $x=a \bar{a} \in D$ such that $X \Longrightarrow_{G} a N_{1} \Longrightarrow_{G} a \bar{a} Y$ with $N_{1} \in N$. For the production $X \rightarrow a N_{1} \in P$, by the construction of $L(X: Y), X \bar{X} a \in A(X)$ and $\bar{X} a N_{1}, a N_{1} \bar{N}_{1} \in C$ hold. Similarly, for the production $N_{1} \rightarrow \bar{a} Y \in P, \bar{a} Y \bar{Y} \in B(Y)$ and $\bar{N}_{1} \bar{a} Y, N_{1} \bar{N}_{1} \bar{a} \in C$ hold.

Then a string $y=X \bar{X} a N_{1} \bar{N}_{1} \bar{a} Y \bar{Y}$ satisfies that $y \in L(X$ : $Y)$ and $h^{\prime}(y)=h^{\prime}(a \bar{a})=h(a \bar{a})=h(x)$.

Induction step: We suppose that the claim holds for any $k \leq n$. Consider a string $x \in D$ which satisfies that $X \Longrightarrow_{G}^{*} x Y$ and $|x|=2(n+1)$. Since $x$ is in $D$, there are the following two cases for $x$ :

1. $x=\alpha \beta$, where $\alpha, \beta \in D$ and $2 \leq|\alpha|,|\beta| \leq n$.

Let $N_{1}$ be a nonterminal symbol in $N$ such that $X \Longrightarrow_{G}^{*}$ $\alpha N_{1} \Longrightarrow_{G}^{*} \alpha \beta Y$.

For the string $\alpha \in D$ satisfying that $X \Longrightarrow_{G}^{*} \alpha N_{1}$ and $2 \leq|\alpha| \leq n$, from the induction hypothesis, there is a string $y_{1}$ such that $y_{1}=X \bar{X} z_{1} N_{1} \bar{N}_{1} \in L\left(X: N_{1}\right) \cap D^{\prime}$ with $z_{1} \in D^{\prime}$ and $h(\alpha)=h^{\prime}\left(y_{1}\right)$. Similarly for $\beta$, there is a string $y_{2}$ such that $y_{2}=N_{1} \bar{N}_{1} z_{2} Y \bar{Y} \in L\left(N_{1}: Y\right) \cap D^{\prime}$ with $z_{2} \in D^{\prime}$ and $h(\beta)=h^{\prime}\left(y_{2}\right)$.

Consider a string $y=X \bar{X} z_{1} N_{1} \bar{N}_{1} z_{2} Y \bar{Y}$. From the fact that $A\left(N_{1}\right), B\left(N_{1}\right) \subset C, y$ is in $L(X: Y)$. Since $z_{1}$ and $z_{2}$ are in $D^{\prime}, y$ is in $D^{\prime}$. Further,

$$
\begin{aligned}
h^{\prime}(y) & =h^{\prime}\left(X \bar{X} z_{1} N_{1} \bar{N}_{\left.1 z_{2} Y \bar{Y}\right)}\right. \\
& =h^{\prime}\left(X \bar{X} z_{1} N_{1} \bar{N}_{1} N_{1} \bar{N}_{1} z_{2} Y \bar{Y}\right) \\
& =h^{\prime}\left(y_{1}\right) h^{\prime}\left(y_{2}\right)=h(\alpha) h(\beta)=h(\alpha \beta) .
\end{aligned}
$$

2. $x=a \alpha \bar{a}$, where $\alpha \in D, a \in \Sigma, \bar{a} \in \bar{\Sigma}$, and $|\alpha|=2 n$.

Let $N_{1}, N_{2}$ be nonterminal symbols in $N$ such that $X \Longrightarrow_{G} a N_{1} \Longrightarrow_{G}^{*} a \alpha N_{2} \Longrightarrow_{G} a \alpha \bar{a} Y$.

For the string $\alpha$, from the induction hypothesis, there is a string $y_{1}=N_{1} \bar{N}_{1} z_{1} N_{2} \bar{N}_{2}$ such that $y_{1} \in D^{\prime}, h(\alpha)=$ $h^{\prime}\left(y_{1}\right)$, and $y_{1} \in L\left(N_{1}: N_{2}\right)$.

For the production rule $X \rightarrow a N_{1}$ in $P$, by the construction of $L(X: Y), X \bar{X} a \in A(X)$ and $\bar{X} a N_{1}, a N_{1} \bar{N}_{1} \in C$ hold. Similarly, for the production rule $N_{2} \rightarrow \bar{a} Y$ in $P, N_{2} \bar{N}_{2} \bar{a}, \bar{N}_{2} \bar{a} Y \in C$ and $a Y \bar{Y} \in B(Y)$ hold. From the fact that $A\left(N_{1}\right), B\left(N_{2}\right) \subset C$, a string $y=X \bar{X} a y_{1} \bar{a} Y \bar{Y}=$ $X \bar{X} a N_{1} \bar{N}_{1} z_{1} N_{2} \bar{N}_{2} \bar{a} Y \bar{Y}$ is in $L(X: Y)$. Since $z_{1}$ is in $D^{\prime}$, $y$ is in $D^{\prime}$. Further,

$$
\begin{aligned}
h^{\prime}(y) & =h^{\prime}\left(X \bar{X} a y_{1} \bar{a} Y \bar{Y}\right) \\
& =h^{\prime}(X \bar{X} a) h^{\prime}\left(y_{1}\right) h^{\prime}(a Y \bar{Y}) \\
& =h(a) h(\alpha) h(\bar{a})=h(a \alpha \bar{a}) .
\end{aligned}
$$

The inclusion $h(D \cap L(G)) \subseteq h^{\prime}\left(D^{\prime} \cap L(S: F)\right)$ can be proved by considering the case $X=S$ and $Y=F$ in the previous claim.

Note that if $\lambda$ is in $D \cap L(G)$, then $S \bar{S} F \in A(S: F)$ and $S F \bar{F} \in B(S: F)$ hold. The string $S \bar{S} F \bar{F}$ satisfies $S \bar{S} F \bar{F} \in$ $L(S: F) \cap D^{\prime}$ and $h^{\prime}(S \bar{S} F \bar{F})=\lambda=h(\lambda)$.

$\left[\boldsymbol{h}(D \cap \boldsymbol{L}(\boldsymbol{G})) \supseteq \boldsymbol{h}^{\prime}\left(D^{\prime} \cap \boldsymbol{L}(\boldsymbol{S}: \boldsymbol{F})\right)\right]$ We will prove the converse inclusion, starting by showing that for a string $y \in$ $D^{\prime} \cap L(X: Y)$ with $X, Y \in N$, there is a string $x$ such that $x \in D, X \Longrightarrow_{G}^{*} x Y$, and $h(x)=h^{\prime}(y)$.

For the string $y \in D^{\prime} \cap L(X: Y)$ such that $|y|_{T}=2 n$, we will prove the claim by induction on $n$.

Base step: Consider a string $y=X \bar{X} a N_{1} \bar{N}_{1} \bar{a} Y \bar{Y}$ in $D^{\prime} \cap L(X: Y)$. From the fact $\bar{X} a N_{1}, \bar{N}_{1} \bar{a} Y \in C$, production rules $X \rightarrow a N_{1}$ and $N_{1} \rightarrow \bar{a} Y$ are in $P$. For the string $x=a \bar{a}$, there is a derivation $X \Longrightarrow_{G} a N_{1} \Longrightarrow_{G} a \bar{a} Y$ and $h(x)=$ $h^{\prime}\left(X \bar{X} a N_{1} \bar{N}_{1} \bar{a} Y \bar{Y}\right)=h^{\prime}(y)$.

Induction step: We suppose that the claim holds for any $k \leq n$. Consider a string $y \in D^{\prime} \cap L(X: Y)$ which satisfies that $|y|_{T}=2(n+1)$. There are the following cases for $y$.

- $y=\alpha \beta$, where

$$
\begin{aligned}
& \alpha=X \bar{X} a_{1} N_{1} \bar{N}_{1} a_{2} N_{2} \bar{N}_{2} \cdots a_{2 i} N_{2 i} \bar{N}_{2 i} \in D^{\prime}, \\
& \beta=b_{1} N_{2 i+1} \bar{N}_{2 i+1} b_{2} N_{2 i+2} \bar{N}_{2 i+2} \cdots b_{2 j} Y \bar{Y} \in D^{\prime},
\end{aligned}
$$

with $i, j \geq 1$ and $i+j=n+1$.

From the induction hypothesis, for the string $\alpha$ in $D^{\prime} \cap$ $L\left(X: N_{2 i}\right)$, there is a string $x_{1} \in D$ such that $X \Longrightarrow_{G}^{*}$ $x_{1} N_{2 i}$ and $h\left(x_{1}\right)=h^{\prime}(\alpha)$.

For the string $\beta$ in $D^{\prime}$, consider a string $\beta^{\prime}=N_{2 i} \bar{N}_{2 i} \beta$ in $D^{\prime}$. From the induction hypothesis for $\beta^{\prime} \in L\left(N_{2 i}: Y\right) \cap$ $D^{\prime}$, there is a string $x_{2}$ such that $x_{2} \in D, N_{2 i} \Longrightarrow_{G}^{*} x_{2} Y$ and $h\left(x_{2}\right)=h^{\prime}\left(\beta^{\prime}\right)$.

From the fact that $x_{1}, x_{2} \in D$, the string $x_{1} x_{2}$ is in $D$, which satisfies that $X \Longrightarrow_{G}^{*} x_{1} N_{2 i} \Longrightarrow_{G}^{*} x_{1} x_{2} Y$ and $h\left(x_{1} x_{2}\right)=h\left(x_{1}\right) h\left(x_{2}\right)=h^{\prime}(\alpha) h^{\prime}\left(\beta^{\prime}\right)=h^{\prime}(\alpha) h^{\prime}(\beta)=$ $h^{\prime}(\alpha \beta)$.

- $y=\alpha \beta$, where

$$
\begin{aligned}
& \alpha=X \bar{X} a_{1} N_{1} \bar{N}_{1} a_{2} N_{2} \bar{N}_{2} \cdots a_{2 i-1} N_{2 i-1} \bar{N}_{2 i-1} \in D^{\prime}, \\
& \beta=N_{2 i} \bar{N}_{2 i} b_{1} N_{2 i+1} \bar{N}_{2 i+1} b_{2} \cdots b_{2 j} Y \bar{Y} \in D^{\prime},
\end{aligned}
$$

with $i, j \geq 1$ and $i+j=n+1$.

In a similar way to the case $y=\alpha \beta$, where $\alpha=X \bar{X} a_{1} N_{1} \bar{N}_{1} a_{2} N_{2} \bar{N}_{2} \cdots a_{2 i} N_{2 i} \bar{N}_{2 i}$ and $\beta=$ $b_{1} N_{2 i+1} \bar{N}_{2 i+1} b_{2} N_{2 i+2} \bar{N}_{2 i+2} \cdots b_{2 j} Y \bar{Y}$, we can prove the existence of a string $x$ such that $x \in D, h(x)=h^{\prime}(\alpha \beta)$, and $X \Longrightarrow_{G}^{*} x Y$.

- $y=X \bar{X} a_{1} z a_{2 n+2} Y \bar{Y}$, where $z=N_{1} \bar{N}_{1} a_{2} \cdots a_{2 n+1}$ $N_{2 n+1} \bar{N}_{2 n+1} \in D^{\prime}, Y=X$ and $a_{2 n+2}=\bar{a}_{1}$.

For the string $z \in D^{\prime} \cap L\left(N_{1}: N_{2 n+1}\right)$, from the induction hypothesis, there is a string $x_{1}$ such that $x_{1} \in D$, 
$N_{1} \Longrightarrow{ }_{G}^{*} x_{1} N_{2 n+1}$, and $h\left(x_{1}\right)=h^{\prime}(z)$.

From the strings $\bar{X} a_{1} N_{1}$ and $\bar{N}_{2 n+1} \bar{a}_{1} X$ in $C$, there are production rules $X \rightarrow a_{1} N_{1}$ and $N_{2 n+1} \rightarrow \bar{a}_{1} X$ in $P$. Therefore, $X \Longrightarrow_{G} a_{1} N_{1} \Longrightarrow_{G}^{*} a_{1} x_{1} N_{2 n+1} \Longrightarrow_{G}$ $a_{1} x_{1} \bar{a}_{1} X$ holds. Further, the string $a_{1} x_{1} \bar{a}_{1}$ satisfies that $h\left(a_{1} x_{1} \bar{a}_{1}\right)=h\left(X \bar{X} a_{1} z \bar{a}_{1} Y \bar{Y}\right)=h(y)$ and $a_{1} x_{1} \bar{a}_{1} \in D$.

The inclusion $h(D \cap L(G)) \supseteq h^{\prime}\left(D^{\prime} \cap L(S: F)\right)$ can be proved by considering the case $X=S$ and $Y=F$ in the previous claim.

Note that if $S \bar{S} F \bar{F} \in D^{\prime} \cap L(S: F)$, then for the string $S \bar{S} F \in A(S: F)$, by the construction of $L(S: F)$, there is a production $S \rightarrow \lambda$ in $P$, which implies that $\lambda \in D \cap L(G)$. Further, $h^{\prime}(S \bar{S} F \bar{F})=\lambda=h(\lambda)$ holds.

Since the class of context-free languages is closed under intersection with regular languages and morphisms, $H($ Dyck $\cap L O C(3)) \subseteq C F$ holds from LOC(3) $\subset R E G$ in Theorem 1. Furthermore, from Theorem 4 and ChomskySchützenberger characterization of context-free languages $C F=H(D y c k \cap R E G)$, we have the following theorem.

Theorem 5: $C F=H(D y c k \cap L O C(3))$.

From the definition of Dyck language, we can easily show that for any Dyck language $D$, there is an insertion system $\gamma$ of weight $(2,0)$ which satisfies that $D=L(\gamma)$. Therefore, the next result follows from the fact Dyck $\subset I N S_{2}^{0} \subset$ $C F$ and Theorem 5.

Note 1: $C F=H\left(I N S_{2}^{0} \cap \operatorname{LOC}(3)\right)$.

Furthermore, from Theorem 2 and the fact that for arbitrary $k$ with $k \geq 1$, the class of regular languages includes the class of strictly $k$-testable languages, we have the following corollary.

Corollary 2: $C F=H($ Dyck $\cap \operatorname{LOC}(k))(k \geq 3)$. $C F=H\left(I N S_{i}^{0} \cap \operatorname{LOC}(k)\right)(i \geq 2, k \geq 3)$.

\section{Conclusion}

In this paper, we have contributed to the study of insertion systems with new characterizations of context-free languages. Specifically, we have shown that $C F=H($ Dyck $\cap$ $\operatorname{LOC}(k))(k \geq 3)$ and $C F=H\left(I N S_{i}^{0} \cap \operatorname{LOC}(k)\right)(i \geq 2, k \geq$ 3).

The following characterizations of regular languages in terms of insertion languages and strictly locally testable languages have shown in [8].

- $H\left(I N S_{1}^{0} \cap L O C(1)\right) \subset R E G$.

- $R E G=H\left(I N S_{1}^{0} \cap L O C(k)\right)(k \geq 2)$.

- $R E G$ and $H\left(I N S_{i}^{0} \cap L O C(1)\right)$ are incomparable $(i \geq 2)$.

- $R E G \subset H\left(I N S_{i}^{0} \cap \operatorname{LOC}(k)\right)(i \geq 2, k \geq 2)$.

The followings are open problems:

- Can $C F$ be represented as $C F=H\left(I N S_{2}^{0} \cap L O C(2)\right)$ ? This is useful to show that the value of parameter $k=3$ in the strictly $k$-testable languages in Note 1 is necessary for expressing context-free languages, or not.
- Can $C F$ be represented as $C F=H\left(I N S_{i}^{j} \cap L O C(k)\right)$ for $i, j \geq 1$ and $k \geq 1$ ?

This is useful to see the difference between insertion systems with context-checking and context-free insertion systems.

\section{Acknowledgements}

The author is deeply indebted to T.Yokomori for his helpful discussions. She is also deeply indebted to referees whose invaluable comments improved the draft of this paper. This work is supported in part by Grant-in-Aid for the Research Institute for Science and Technology of Tokyo Denki University with no.Q07J-05.

\section{References}

[1] S. Verlan, "On minimal context-free insertion-deletion systems," J. Autom. Lang. Combin., vol.12, no.1, pp.317-328, 2007.

[2] G. Păun, G. Rozenberg, and A. Salomaa, DNA Computing. New Computing Paradigms, Springer, 1998.

[3] K. Onodera, "A note on homomorphic representation of recursively enumerable languages with insertion grammars" IPSJ J., vol.44, no.5, pp.1424-1427, 2003.

[4] G. Păun, M.J. Pérez-Jiménez, and T. Yokomori, "Representations and characterizations of languages in Chomsky hierarchy by means of insertion-deletion systems" Int. J. Found. Comput. Sci., vol.19, no.4, pp.859-871, 2008.

[5] T. Head, "Splicing representations of strictly locally testable languages," Discrete Appl. Math., vol.87, pp.87-139, 1998.

[6] G. Rozenberg and A. Salomaa, eds., Handbook of formal languages, Springer-Verlag New York, New York, NY, USA, 1997.

[7] R. McNaughton and S.A. Papert, Counter-Free Automata (M.I.T. research monograph no. 65), The MIT Press, 1971.

[8] K. Onodera, "New morphic characterizations of languages in chomsky hierarchy using insertion and locality," Lect. Notes Comput. Sci., pp.648-659, 2009.

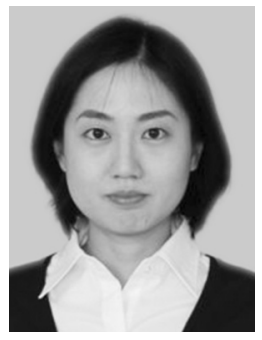

Kaoru Fujioka was born in Tokyo, Japan. Her maiden name is Kaoru Onodera. She received B.S., M.S. and Ph.D degrees from Waseda University, Tokyo, Japan, in 2000, 2002, and 2007 respectively. Since 2009, she has been a Research Assistant Professor in the Office for Strategic Research Planning, Kyushu University, Fukuoka, Japan. Her research interests include DNA computing, computational learning theory, and formal language theory. She is a member of IPSJ. 